Eur Psychiatry (1993) 8, 5, 233-234

(C) Elsevier, Paris

Editorial

\title{
The importance of national therapeutic strategies for the treatment of sub-threshold depressive disorders
}

\author{
Y Lecrubier \\ Hôpital de la Salpêtrière, Pavillon Clérambault, U302, 47, boulevard de l'Hôpital, 75651 Paris Cedex 13, France
}

Depression and anxiety are the commonest disorders of all psychiatric diagnosis making up $79 \%$ of all diagnoses (Sartorius and Harding, 1983).

The usual reported rate for depression in primary care, for those patients with an access to care, is 10 to $20 \%$ in Western as well as in developing countries (Orley and Wing, 1979).

This refers to well identified depressive disorders according to international diagnosis criteria as proposed by ICD 10 or DSM III or IV. Additionally, it should be stressed that our knowledge concerning the natural history and the classification of depressive disorders suffers from difficulties.

The last WHO epidemiological study in primary care performed in 14 countries shows that current diagnosis of depression in primary care is found in more than $10 \%$ of patients (Sartorius et al, 1993). In addition, $8 \%$ suffer from sub-threshold disorders.

These patients with ill-defined depressive syndromes are considerably disabled. Moreover, they are treated by their general practitioner as frequently as patients with well-defined depression.

Depression is among the disorders and illnesses inducing the higher social impact and impairment with high treatment rates, low working abilities and high impairment of social and leisure activities. The depressed with minor and moderate syndromes are found to be substantially impaired with a high health service consumption and a high suicidal rate similar to those encountered in major depression.

In addition to the personal and socio-economic cost, depression is associated with an increased mortality. Suicide rates among treated depressives are as high as 12 to 25\%. Mortality is also increased for somatic reasons especially from cardiovascular disorders as shown in the Community Oxford Record Linkage study (Baldwin, 1980) and the Danish study of Weeke et al (1987).

The figures are very similar to those observed in major depression and these patients should therefore be taken into consideration and treated as well as more severely depressed patients.

Four possibilities exist to classify subthreshold depressed patients:

- the syndrome and the course of illness is similar to that of major depression but the symptomatic severity is too low to reach the criteria for MDE (Major Depressive Episode). 
- a chronic mild depressive syndrome probably close to the diagnosis of neurasthenia proposed by ICD 10 , is a disabling condition due to chronicity.

- severe episodes with a duration too short to reach the criteria proposed by DSM or ICD 10, led to the definition of recurrent brief depression as proposed by Angst. Social impairment and suicide rates are high in this population.

- cross sectional syndromes observed in patients reaching or not diagnostic criteria may reflect a specific psychopathology and it is claimed that these patients need specific treatments. This is the case for "atypical depression" as defined by Liebovwitz et al or even for neurasthenia or thymasthenia.

In the absence of well-defined diagnostic criteria for this very common group of patients, most countries developed diagnostic patterns and local treatment strategies.

The following papers* will describe the heterogeneous treatment strategy and diagnostic concepts for Asian, South-American, North-American and European countries.

This set of papers provides a contribution to a better understanding of what is considered to be depression in the world, and describes the various possibilities, adopted worldwide to treat a group of patients as large as $10 \%$ of those with access to care.

\section{References}

Baldwin JA (1980) Schizophrenia and physical disease: a preliminary analysis of the data from the Oxford Record Linkage Study. In: Biochemistry of Schizophrenia and Addiction. In Search of a Common Factor (Hemmings G, ed). MTP Press, Lancaster, 297-318

Orley J, Wing J (1979) Psychiatric disorders in two African villages. Arch Gen Psychiatry 36, 513-520

Sartorius N et al (1993) An international study of psychopathological problems in primary care: preliminary report from the WHO collaborative project on psychological problems in general health care (in press)

Sartorius N, Harding TW (1983) The WHO collaborative study on strategies for extending mental health care. I. The genesis of the study. Arch Gen Psychiatry 140, 1470-1473

Weeke A, Juel K, Vaeth M (1987) Cardiovascular death and manic-depressive psychosis. J Affective Disord 13, $287-292$

* These papers were presented during the symposium on "Worldwide therapeutic strategies in atypical depressive syndromes" organised by the World Psychiatric Association section of pharmacology, within the framework of the 9th World Congress of Psychiatry (Rio de Janeiro, June 1993).

We acknowledge Synthelabo, International Division, for their support in the organisation of this symposium. 